\title{
Septic Cerebral Vein Thrombosis and Abnormal Leptomeningitis as a Complication From a Periodontitis in a Hispanic Male: Case Report and Literature Review
}

\author{
Sarmad Said ${ }^{\mathrm{a}, \mathrm{b}}$, Chad J. Cooper ${ }^{\mathrm{a}}$, Haider Alkhateeb ${ }^{\mathrm{a}}$, Sucheta Gosavi ${ }^{\mathrm{a}}$, Ogechika Alozie ${ }^{\mathrm{a}}$
}

\begin{abstract}
Sinus vein thrombosis is a rare form of stroke that results from thrombosis of the dural vein sinus. Despite the variety of diagnostic procedures and treatment, septic thrombosis of the cavernous sinus can cause significant morbidity and mortality if not diagnosed and treated as an emergency. The clinical presentation of proptosis that occurs suddenly accompanied by erythema of the eyelid and limitation of ocular movement should alert the clinician of the possibility of cavernous sinus thrombosis. A 38-year old Hispanic male presented with pounding headache, mainly in the occipital region that began 1 week prior to hospital admission. During the hospitalization the patient subsequently developed a progressive worsening of proptosis, lacrimation and neck pain. The MRI and venography of the brain showed a cerebral venous thrombosis (CVT) with a reactive diffuse leptomeningitis. The underlying cause was a chronic dental infection. The patient was treated with course of antibiotics and discharged. A few weeks later the repeated imaging studies showed a resolution of the CVT and leptomeningitis. Although rare, septic vein thrombosis (SVT) remains a potentially lethal complication of infections that involve the sinuses, face, ears and oral cavity. The early recognition and differentiation from other diseases are keys to reducing mortality rates and long-term sequelae. Advances in imaging, especially CT and MRI have contributed substantially to the rapid diagnosis of the condition.
\end{abstract}

Keywords: Periodontitis; Leptomeningitis; Cerebral vein thrombosis

\footnotetext{
Manuscript accepted for publication July 5, 2013

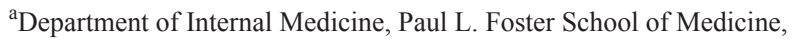
Texas Tech University Health Sciences Center, 4800 Alberta Ave, El Paso, Texas, 79905, USA

${ }^{\mathrm{b}}$ Corresponding author: Sarmad Said, Department of Internal Medicine, Paul L. Foster School of Medicine, Texas Tech University Health Sciences Center, 4800 Alberta Ave, El Paso, Texas, 79905, USA. Email: sarmad.said@ttuhsc.edu
}

doi: http://dx.doi.org/10.4021/jmc1370w

\section{Introduction}

Cerebral venous thrombosis (CVT) is an uncommon cerebrovascular disease presenting with a remarkably wide spectrum of signs and mode onset [1]. CVT may occur as a complication of infectious or noninfectious processes. Septic thrombosis describes a thrombophlebitic process affecting the cavernous sinus with an infective etiology. Reviews of large single center series from the pre-antibiotic and early antibiotic era had documented that infections of the middle third of the face were responsible for most cases of SVT. Approximately 60 to $80 \%$ of these nasal furunculosis accounts for the most common cause [2]. Organisms may reach the cavernous sinus from the face by an anterograde route along the ophthalmic veins, which is connected to the angular veins, or by a retrograde route along the emissary veins that are connected to the pterygoid venous plexus. In up to 25 per cent of cases where a facial furuncle is responsible, it has either been previously manipulated by the patient or incised by the surgeon [3]. Staphylococcus aureus is the most frequently cultured organism in these infections accounting for $70 \%$, followed by Streptococcus species at $20 \%$ [4].

Septic thrombosis of the dural venous sinuses is a rare but often catastrophic complication by a variety of infectious processes. Bacterial meningitis and paranasal sinusitis can be a complication of superior sagittal thrombosis, resulting in an $80 \%$ mortality rate [5].

Septic CVT can occur at any age but typically affects young adults, most commonly in the 3 rd decade of life. The incidence is $3-4 / 1,000,000$ with 3:1 female predominance [6].

Early diagnosis can be facilitated by prompt recognition of the clinical and radiological findings that are suggestive of venous occlusion of the cavernous sinus. The vast majority of cases of septic CVT have an acute presentation associated with prominent features of sepsis. The latent period between symptoms of the predisposing infection and the clinical features of septic SVT varies between one and twenty-one days, but averaged about five to six days in one series [3]. Pyrexia occurs in over 90 per cent of cases, which is usually severe, and may have a 'picket fence' pattern suggestive of throm- 

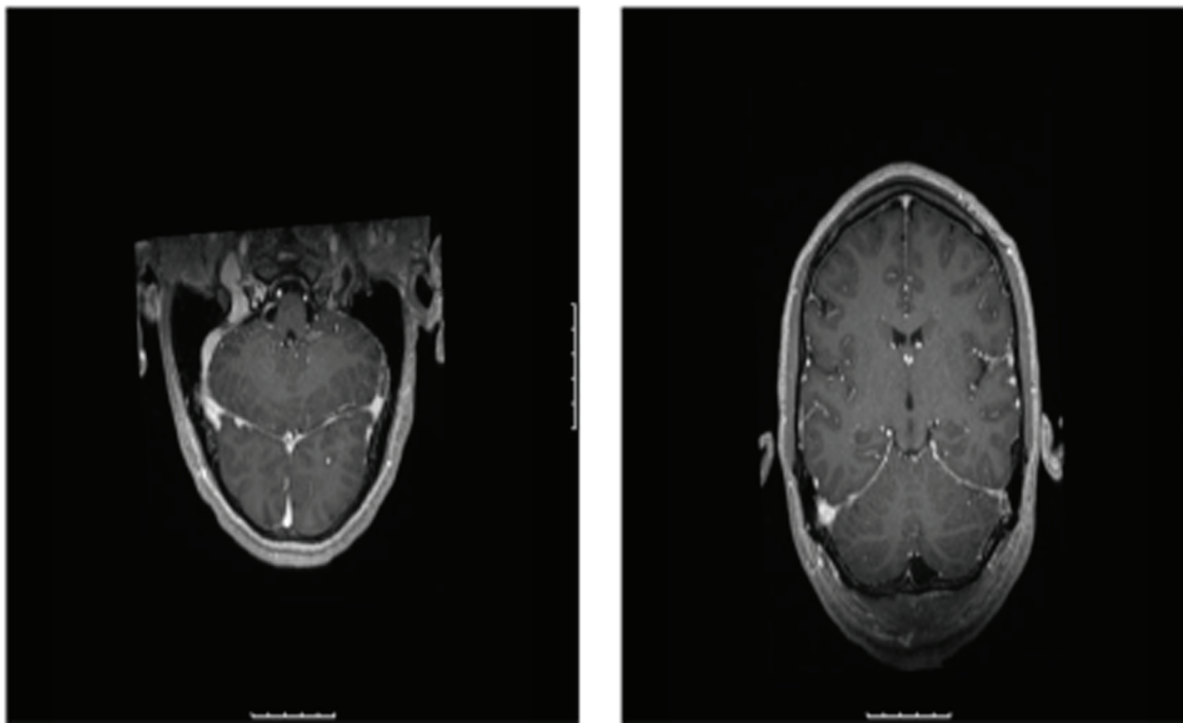

Figure 1. Diffuse supra and infratentorial leptomeningitis and acute thrombosis of the left cavernous sinus, superior petrosal sinus, sigmoid sinus, jugular bulb, and suboccipital vein.

bophlebitis. Other features of sepsis may be the presence of tachycardia, vomiting, hypotension, confusion, rigors and comatose [3]. Headache has been reported in about $52-90$ percent and is typically unilateral, with a retro-orbital or frontotemporal area distribution [7].

Impaired vision is uncommon. Nevertheless, visual loss may be caused by corneal ulceration secondary to proptosis and loss of the corneal reflex, occlusion of the internal carotid, ischemic optic neuropathy, orbital congestion, toxic neuritis of the optic nerve, or embolic phenomena [8].

$\mathrm{CT}$ and MRI are the primary radiological modalities used to confirm the diagnosis if suspected on clinical grounds. The most sensitive investigation of intracavernous occlusive defects is venography. Nowadays, cerebral angiography has been reserved for the definitive assessment of intracavernous aneurysms after they have been detected and monit $\neg$ ored using CT or MRI [9].

Undoubtedly, antibiotics have the greatest impact on the prognosis of SVT [10]. Postmortem studies have shown that there is less extensive thrombosis within the cavernous sinuses since the advent of antibiotics [10].

We present a case of septic cavernous sinus thrombosis as a complication of a leptomeningitis, which resulted from a periodontitis in a Hispanic male patient.

\section{Case Report}

38 years old right handed, Hispanic male without a significant past medical history presented to the ER with a progressive pounding occipital headache that started almost 1 week prior to admission. Approximately 2 weeks prior to admission, he visited his primary care physician in Juarez,
Mexico and was given amoxicillin for an unknown reason. Four days before the admission he visited the ER in another hospital and was given NSAIDs and diazepam and was discharged home. Over the following two days before the admission he experienced progressive lacrimation, eye discomfort aggravated by eye movement and minimal swelling around his left eye. He described subjective febrile episodes. He denied chest pain or shortness of breath. Social history was negative for tobacco, alcohol or drug use. No pertinent physical findings noted except minimal left eye proptosis and lacrimation. Neurological exam revealed a minimally positive Kernig sign. The laboratory findings were positive for a leukocytosis of 14.81, but otherwise other lab values were unremarkable.

A computer-tomography of the brain was done in the emergency department. No acute intracranial trauma was noted. Multiple attempts to perform a lumbar puncture in the ER were unsuccessful. Due to the clinical presentation, he was started on empiric antibiotics of vancomycin and ceftriaxone, and an antiviral regimen of acyclovir. A lumbar puncture (LP) was ordered and Interventional Radiology (IR) planned to do this the following day. The next day, it was noted that there was a progressive bulging and swelling of the left eye. On the following day at about 7 A.M. he was unable to open his left eye; at about 11 A.M. he complained about double vision. At this time the neck pain and headache were constant.

The lumbar puncture performed by IR showed WBC of $631 / \mu \mathrm{L}, 68 \%$ segs, $3 \%$ bands, $20 \%$ lymphocytes, $9 \%$ mono-

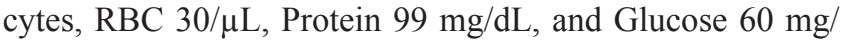
$\mathrm{dL}$. At this time, the differential diagnosis included meningitis, cavernous sinus thrombosis or a retro-orbital abscess. Magnetic resonance imaging of brain showed diffuse supra 

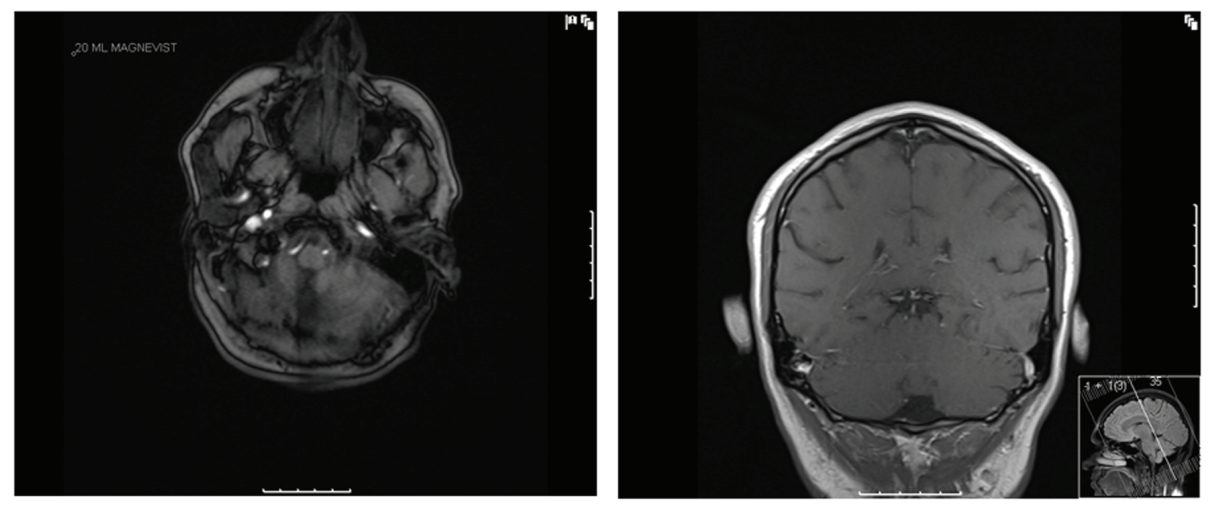

Figure 2. Resolution of abnormal diffuse supra/infratentorial smooth leptomeningeal enhancement. Continued absent flow void and central flow void of left transverse, sigmoid, and jugular vein.

and infratentorial leptomeningitis with acute thrombosis of the left cavernous sinus, superior petrosal sinus, sigmoid sinus, jugular bulb and suboccipital vein (Fig. 1). It was negative for venous infarct, intracranial hemorrhage, midline shift or hydrocephalus. Facial CT was performed and showed a periodontitis in tooth \#17 and \#18 without destruction of the mandibular alveolar septa and without periodontal abscess.

On the 3rd day of hospitalization the antiviral treatment was discontinued, then metronidazole and dexamethasone were added. The microbiological work up was negative for West nile virus, HSV, cryptococcus antigen, group B streptococcus, haemophilus B, streptococcus pneumoniae, neisseria meningitis. On the following day he noticed a remarkable improvement in his headache and neck pain and marked regression of the left eye swelling.

A peripheral inserted central line was placed on the 8th day and the patient was subsequently discharged on metronidazole for two more weeks and instructed to follow up with a dentist immediately to treat his periodontitis, which was determined to be the most likely cause of the cerebral vein thrombosis.

3 weeks after the discharge the patient was presented for a follow up MRI of the brain which showed a resolution of meningitis and the abnormal leptomeningeal enhancement compared to September 18, 2012 as well as resolution of left cavernous sinus thrombosis with continued left transverse, sigmoid, and jugular vein thrombosis (Fig. 2).

\section{Discussion}

CVS is associated with significant mortality, which is estimated at $30 \%$ but can range from $14-79 \%$ according to some case reviews. Approximately half of patients will suffer a residual morbidity, typically from associated cranial neuropathies [11]. In general infection may spread within veins from other sources to the cavernous sinuses as a contiguous phlebitis or thrombophlebitis or even as septic emboli that become trapped by the numerous trabeculations within the cavernous sinus [12].

Oral and dental infections were implicated in less than 10 percent of cases of septic CVT in the early antibiotic era, but are now rare [13]. Infections may spread from the maxillary molar teeth to then enter the orbit via the inferior orbital fissure and then spread into the cavernous sinus. However, a variable character and distribution of headache can occur, which reflects its numerous causes. Nuchal rigidity, which was mild in our patient, has been found in as many as $40 \%$ of patients with CVT and may be due to concurrent bacterial meningitis or meningeal irritation [2]. CVT and the inciting infection are frequently complicated by other intracranial infections including meningitis, encephalitis, brain abscess, pituitary infection, epidural abscess and subdural empyema $[2,3]$.

Diagnosis of cavernous sinus thrombosis is usually made with MRI scan with venogram; fine-cut CT scan is less sensitive [5]. Cerebral angiography with late venous views is the gold standard in the diagnosis of CVT. Because of the morbidity and poor patient acceptance, this technique should only be performed in cases where the diagnosis is doubtful after MRI. However, MRI still remains the routine standard of diagnostic care. CSF cultures may be positive if there is coexistent meningitis. Empirical antimicrobial therapy will depend on the antecedent clinical condition, but should always include an anti-staphylococcal agent. An empirical combination, such as parenteral metronidazole, vancomycin and ceftriaxone will achieve a reasonable proportion of $\mathrm{CSF}$ and brain penetration against $\mathrm{S}$. aureus (including CAMRSA strains) as well as the usual sinus pathogens.

The role and duration of anticoagulation in the management of cavernous sinus thrombosis are controversial. Anticoagulation is most beneficial if given early, but is contraindicated in hemorrhagic intracranial complications [5]. The duration of anticoagulation has varied in several reports from two weeks to several months [14].

Treatment of septic CVT must be prompt and targeted. 
The primary infection must be aggressively drained and the underlying cause eradicated. High-dose intravenous antibiotics that cross the blood-brain barrier and are empirically effective against coagulase-positive staphylococcus and streptococcus are indicated. Nafcillin, third-gener- ation cephalosporins such as cefotaxime, and chloramphenicol are appropriate, pending culture and sensitivity results.

Other supportive treatments that may be necessary in septic CVT include fluid resuscitation, proper eye care, and oxygen. Steroids may be essential in the acute setting to prevent Addisonian crisis [15] as well as in replacement doses in the long-term. However, excluding this indication and the occasional cases where cerebral edema has developed, the role of steroids in septic CVT is contentious. Steroids are potentially harmful because their immunosuppressive effects may accelerate sepsis, but this option must be weighed against the potential benefit of reducing cranial nerve dysfunction or orbital inflammation [16].

In the differential diagnostic setting of SVT an early recognition of the clinical presentation, abrupt management with effective broad-spectrum antibiotics and supportive therapy are essential for full or a significant partial recovery. The primary source of sepsis may be a distant focus with septicemia preceding thrombosis of the cavernous sinus. Alternatively, infection may spread from other sources, i.e. facial regions via the facial venous plexus or from the sphenoid sinus, or as in our patient from a dental infection, directly to the adjacent cavernous sinus.

\section{References}

1. Bousser MG, Ross Russell R. Cerebral venous thrombosis. Vol 1. London: WB Saunders, 1997.

2. Southwick FS, Richardson EP, Jr., Swartz MN. Septic thrombosis of the dural venous sinuses. Medicine (Baltimore). 1986;65(2):82-106.

3. Shaw RE. Cavernous sinus thrombophlebitis: a review. Br J Surg. 1952;40(159):40-48.

4. Tveteras K, Kristensen S, Dommerby H. Septic cav- ernous and lateral sinus thrombosis: modern diagnostic and therapeutic principles. J Laryngol Otol. 1988;102(10):877-882.

5. Tunkel AR. Subdural empyema, epidural abscess and suppurative intracranial thrombophlebitis. In: Mandell GL, Bennett JE, Dolin R, eds. Mandell, Douglas and Bennett's Principles and Practices of Infectious Diseases, 6th edn. Philadelphia, PA: Elsevier Churchill Livingstone; 2005; 1164-1171.

6. Dentali F, Gianni M, Crowther MA, Ageno W. Natural history of cerebral vein thrombosis: a systematic review. Blood. 2006;108(4):1129-1134.

7. Thatai D, Chandy L, Dhar KL. Septic cavernous sinus thrombophlebitis: a review of 35 cases. J Indian Med Assoc. 1992;90(11):290-292.

8. Geggel HS, Isenberg SJ. Cavernous sinus thrombosis as a cause of unilateral blindness. Ann Ophthalmol. 1982;14(6):569-574.

9. Hurst RW, Choi IS, Persky M, Kupersmith M. Mycotic aneurysms of the intracavernous carotid artery: a case report and review of the literature. Surg Neurol. 1992;37(2):142-146.

10. Yarington CT, Jr. Cavernous sinus thrombosis revisited. Proc R Soc Med. 1977;70(7):456-459.

11. DiNubile MJ. Septic thrombosis of the cavernous sinuses. Arch Neurol. 1988;45(5):567-572.

12. Karlin RJ, Robinson WA. Septic cavernous sinus thrombosis. Ann Emerg Med. 1984;13(6):449-455.

13. Harbour RC, Trobe JD, Ballinger WE. Septic cavernous sinus thrombosis associated with gingivitis and parapharyngeal abscess. Arch Ophthalmol. 1984;102(1):94-97.

14. Doyle KJ, Jackler RK. Otogenic cavernous sinus thrombosis. Otolaryngol Head Neck Surg. 1991;104(6):873877.

15. Gallagher RM, Gross CW, Phillips CD. Suppurative intracranial complications of sinusitis. Laryngoscope. 1998;108(11 Pt 1):1635-1642.

16. Solomon OD, Moses L, Volk M. Steroid therapy in cavernous sinus thrombosis. Am J Ophthalmol. 1962;54:1122-1124. 